\title{
PAPERS
}

\section{Differences in gastric mechanosensory function after repeated ramp distensions in non-consulters with dyspepsia and healthy controls}

\author{
G Holtmann, J Gschossmann, J Neufang-Hüber, G Gerken, N J Talley
}

\begin{abstract}
Background-Abnormal visceral mechanosensory function has been reported in $\mathbf{5 0 \%}$ of non-ulcer (functional) dyspepsia patients. However, only a minority of subjects with functional dyspepsia ever seek medical attention. Whether factors promoting health care seeking behaviour explain visceral hypersensitivity is unknown. Decreased rectal thresholds following sigmoid mechanical stimulation have been observed in irritable bowel but this mechanism has not been evaluated in functional dyspepsia. Aims-To compare visceral mechanosensory function in healthy asymptomatic subjects and non-consulters with chronic unexplained dyspepsia.
\end{abstract}

Methods-Forty two volunteers were recruited: 10 had a history of chronic or recurrent upper abdominal pain or discomfort as assessed by a standardised questionnaire, and Helicobacter pylori status was determined (ELISA and ${ }^{13} \mathrm{C}$ urea breath test). Eight $H$ pylori negative, currently asymptomatic dyspeptic subjects who were non-consulters and eight asymptomatic age and sex matched $H$ pylori negative controls were enrolled. With a barostat bag in the proximal part of the stomach, visceral perception thresholds were determined by random tracking. Thereafter, standardised ramp distensions were performed $(2 \mathrm{~mm} \mathrm{Hg}$ increments, duration of each pressure step 30 seconds, maximum pressure $35 \mathrm{~mm}$ Hg (or occurrence of pain)) and tracking of sensory thresholds and ramp distension repeated every 30 minutes for a total of two hours.

Results-Overall, thresholds for first perception were significantly lower in dyspeptic subjects compared with asymptomatic controls (12.5 (0.6) $\mathrm{mm} \mathrm{Hg} v 17.5(1.0) \mathrm{mm}$ $\mathrm{Hg} ; \mathrm{p}<0.02)$. After repeated ramp distensions, thresholds for first perception significantly increased by $3.6(0.7) \mathrm{mm} \mathrm{Hg}$ in healthy subjects compared with 0.1 (1.4) $\mathrm{mm} \mathrm{Hg}$ in subjects with dyspepsia $(p<0.05$ dyspeptics $v$ controls).

Conclusions-(1) Repeated mechanical stimulation increases visceral sensory thresholds in asymptomatic subjects while thresholds remain unchanged in dyspeptics.
(2) Visceral hyperalgesia occurs in dyspeptic subjects who are not health care seekers. (Gut 2000;47:332-336)

Keywords: functional dyspepsia; visceral hyperalgesia; sensory function; gastrointestinal motility; sensory adaptation

Altered visceral mechanosensory function (hyperalgesia) is believed to play a key role in the development of symptoms in at least a subgroup of patients with otherwise unexplained (functional) abdominal complaints. ${ }^{1-4}$ The mechanisms involved in the development of visceral hypersensitivity are not yet completely elucidated but impaired central downregulation of visceral afferents, ${ }^{56}$ sensitisation by mucosal inflammation, ${ }^{7}$ or altered central processing ${ }^{8}$ may be important. While symptoms of functional gastrointestinal (GI) disorders are extremely prevalent in the population, only a subgroup of patients with these symptoms seek medical attention. Interestingly, patients seeking medical attention are characterised by psychological distress in a high proportion of subjects, ${ }^{910}$ and some have a history of abuse in childhood. ${ }^{11}$ It is conceivable that psychological factors per se may alter visceral perception and this may explain the apparent association between functional GI disorders and visceral hypersensitivity. Human studies evaluating visceral sensory function have been conducted only in healthy controls and patients (that is, subjects who have sought medical care). Whether visceral sensory abnormalities occur in people who do not seek health care is unknown.

Recently it has been shown that mechanical stimulation of the sigmoid lowers sensory thresholds in patients with irritable bowel syndrome (IBS) but not healthy controls. ${ }^{12}$ Whether this also occurs in subjects with functional dyspepsia is unknown.

We hypothesised that visceral sensory abnormalities are central to the pathogenesis of functional dyspepsia regardless of whether or not health care has been sought. We further hypothesised that repeated mechanical (phasic) stimulation would alter sensory thresholds

Abbreviations used in this paper: IBS, irritable bowel syndrome; GI, gastrointestinal.
Accepted for publication 22 February 2000 
differently in healthy controls and subjects with chronic relapsing dyspepsia.

\section{Material and methods}

Forty two volunteers (aged 24-47 years) were recruited for participation in the study by public advertisements on the University of Essen campus. In all subjects, abdominal symptoms were assessed using the bowel disease questionnaire. ${ }^{1314}$ This version of the questionnaire contains 75 items and has been used in previous studies ${ }^{14}{ }^{15}$; it has been shown to have adequate content as well as predictive and construct validity for measurement of GI tract symptoms. ${ }^{16}$ Ten subjects $(23.8 \%$ (95\% CI 12.1-39.4)) reported a history of chronic or relapsing upper abdominal symptoms (that is, discomfort, pain, postprandial fullness, early satiety) without signs of gastro-oesophageal reflux; none was receiving non-steroidal antiinflammatory drugs and all were currently asymptomatic. No subject had ever consulted a physician for evaluation or treatment of dyspepsia. Because Helicobacter pylori associated peptic ulcers are the most important cause of organic dyspepsia, ${ }^{17} \mathrm{H}$ pylori status was determined by a locally validated ELISA (Elias, Freiburg Germany) and the ${ }^{13} \mathrm{C}$ urea breath test; two $H$ pylori positive subjects with symptoms were excluded from the study. Thus eight $H$ pylori negative subjects with a history of dyspeptic symptoms (but no symptoms at the time of the study, aged 24-38 years, four females) and eight subjects from the same population without symptoms, matched for age $( \pm 5$ years) and sex were included for further evaluation.

All subjects had a normal physical examination and no alarm symptoms (for example, no weight loss) and did not report colonic symptoms such as altered bowel habits or pain associated with defecation. Laboratory testing (that is, white and red blood count, sedimentation rate, fasting blood glucose level, liver function tests) was normal in all subjects. To measuring personality traits, we used the validated German version of the personality research form. ${ }^{18}{ }^{19}$ Subjects were told that they were being enrolled into a study that aimed to elucidate the mechanisms involved in the pathogenesis of functional GI disorders.

PROTOCOL

Following approval of the research protocol by the local ethics committee and informed consent, the subjects swallowed an orogastric tube after an overnight fast. The barostat bag with infinite compliance up to a maximum capacity of 1.5 litres was attached to the tube and positioned in the proximal part of the stomach. All subjects were seated in an upright position. The barostat bag with a double lumen tube was connected to the barostat device (ISOBAR 3 device, G\&J Electronics, Ontario, Canada). Thereafter, the minimal distending pressure that allowed recording of changes in intra-abdominal pressure associated with breathing was determined by increasing the pressure of the barostat bag in $1 \mathrm{~mm} \mathrm{Hg}$ increments.
To determine sensory thresholds, a distension procedure was performed. All subjects received standardised instructions. They were told that the balloon in the stomach would be repeatedly inflated and deflated and they were asked to indicate whether or not they actually perceived this distension stimulus by pressing the appropriate button on a key pad after a light signal. With a ramp distension the approximate threshold for first perception was determined. Thereafter, the distension procedure was started with $60 \%$ of the pressure of the expected sensory threshold. In random order, the distension pressure was either maintained or increased in $2 \mathrm{~mm} \mathrm{Hg}$ increments; each pressure step was maintained for 45 seconds. At the end of the 45 second period the light signal requested subjects to press the appropriate button on the key pad. Thereafter, the distending pressure was lowered to $5 \mathrm{~mm} \mathrm{Hg}$ and maintained at this level for 15 seconds. If subjects had not perceived the preceding distension, the pressure was randomly increased to the previous distension pressure or increased $2 \mathrm{~mm} \mathrm{Hg}$ above this level. As soon as the subject reported perception of the stimulus, the pressure of the next distension was randomly maintained or lowered and the subjects again asked whether or not they perceived the distension. Subjects were kept unaware of the pressure level at each step. The threshold for first perception was defined as the distending pressure when, on average, the volunteer's response changed from "no perception" to "distension perceived".

These measurements of the threshold of first perception were repeated every 30 minutes up to 120 minutes. In the interval between two measurements of visceral sensory thresholds, two ramp distensions $(2 \mathrm{~mm} \mathrm{Hg}$ pressure increments, duration of 20 seconds for each pressure step and a maximum of $35 \mathrm{~mm} \mathrm{Hg}$ or report of pain) were performed 15 minutes apart (fig 1).

For calculations of gastric wall tension, the balloon in the stomach was considered to be of spherical shape.$^{20}$ The radius $(\mathrm{R})$ of the bag was estimated from the volume of the sphere, and wall tension was calculated from the estimated bag radius and intragastric pressure $(\mathrm{P})$ using Laplaces's law $(\mathrm{T}=\mathrm{P} \times \mathrm{R} / 2){ }^{20}$

\section{STATISTICAL ANALYSIS}

Mean (SEM) values were calculated. Analysis of variance for repeated measurements was
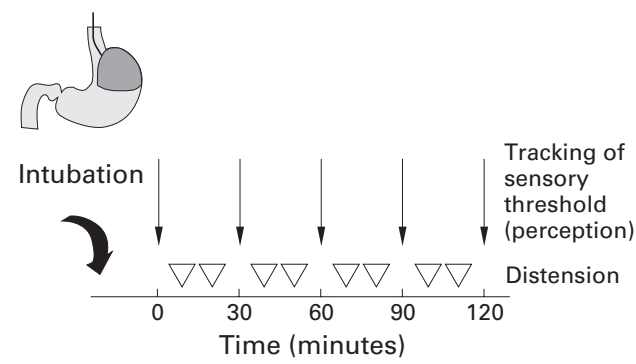

Figure 1 Schematic representation of the experimental design (assessment of sensory thresholds (tracking) and phasic distension (2 $\mathrm{mm} \mathrm{Hg}$ increments, 20 seconds duration)). 


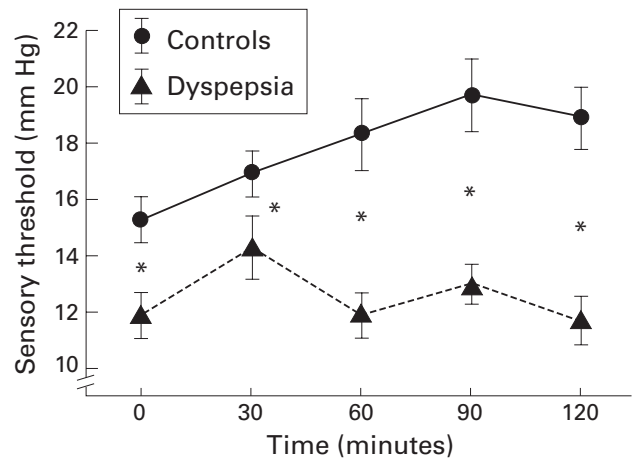

Figure 2 Sensory thresholds for first perception $(\mathrm{mm} \mathrm{Hg}$ ) in controls and in those with a history of dyspepsia. ${ }^{\star} p<0.05$ dyspepsia $v$ controls.

used to compare the main dependent variables in asymptomatic and symptomatic subjects and to determine the effects of the ramp distension in the different groups. All $\mathrm{p}$ values calculated were two tailed; an $\alpha$ level of $5 \%$ was considered significant. Statistical analyses were performed using the Statistical Analysis System. ${ }^{21}$

\section{Results}

MINIMAL DISTENDING PRESSURE

The minimal distending pressure and respective volumes that allowed recording of intraabdominal pressure and volumes changes associated with breathing were $9.5(0.5)$ $\mathrm{mm} \mathrm{Hg}$ and 168 (65) $\mathrm{ml}$ in healthy subjects, and $8.1(0.5) \mathrm{mm} \mathrm{Hg}$ and 209 (79) $\mathrm{ml}$ in subjects with a history of dyspepsia (all $\mathrm{p}>0.4$ ).

TOLERATED RAMP DISTENSIONS

Maximum volumes and pressures applied during ramp distensions were not significantly different for subjects with or without a history of dyspeptic symptoms (volume 845 (88) $\mathrm{ml} v$ 878 (124) $\mathrm{ml}$ and pressure 22.3 (1.8) $v 26$ (0.7) $\mathrm{mm} \mathrm{Hg}$; all $\mathrm{p}>0.4$ ).

PERCEPTION THRESHOLD (TRACKING)

Figure 1 depicts the sensory thresholds (pressure) before and after repeated phasic distensions. Baseline thresholds of first perception and mean thresholds for all repeated measurements of first perception were significantly lower $(\mathrm{p}<0.05)$ in subjects with a history of dyspeptic symptoms compared with controls. In both populations, there was a transient but significant $(\mathrm{p}<0.05)$ increase in sensory thresholds between the first and second tracking of thresholds (fig 2). Thereafter, sensory thresholds decreased in subjects with a history of dyspeptic symptoms while thresholds increased in asymptomatic subjects. There was a significant interaction (group $\times$ time) for the perception threshold (pressure $\mathrm{F}=4.2$, $\mathrm{p}<0.01)$

Similar to the pressure values, mean wall tension was lower in dyspeptics compared with controls (wall tension $0.13(0.01) v 0.18(0.01)$ $\left.\mathrm{mm} \mathrm{Hg} / \mathrm{cm}^{2} ; \mathrm{F}=5.1, \mathrm{p}<0.05\right)$ and the change in tension thresholds mirrored the pressures changes (group $\times$ time $\mathrm{F}=2.9, \mathrm{p}<0.05$ ). Consist- ent with the alterations in pressure and wall tension, volume changes occurred, although these were not significant $(\mathrm{p}=0.08)$.

\section{PERSONALITY TRAITS}

All study subjects (controls and dyspeptics) had personality traits within the normal range of the reference population, and there were no significant differences between controls and dyspeptics (all p>0.20).

\section{Discussion}

The present study identified abnormal sensory function in non-health care seeking subjects with symptoms of functional dyspepsia. In addition, compared with controls, subjects with symptoms of functional dyspepsia were characterised by failure of mechanosensory thresholds for first perception to increase following repeated distensions.

It is now established that lowered sensory thresholds (that is, volumes or pressures that are associated with the first perception of a mechanical stimulation induced by inflating a balloon in the lumen of the gut, or tolerance of a maximal mechanical stimulation) are present in at least a proportion of patients with unexplained dyspepsia (referred to as nonulcer or functional dyspepsia) and IBS. ${ }^{13422} 23$ This has led to the hypothesis that altered mechanosensory function plays a key role in the pathophysiology of functional GI disorders. In contrast, only a minority of subjects with functional GI disorders seek medical attention. Numerous factors are believed to determine health care seeking behaviour, including intensity of symptoms, ${ }^{15}$ personality traits such as anxiety or neuroticism, ${ }^{24}$ and ethnic or sociodemographic factors. ${ }^{25}$ A recent study that performed a follow up of subjects after an infectious episode of diarrhoea observed the development of persistent IBS symptoms in a subset of subjects with psychological abnormalities, such as high neuroticism scores. ${ }^{26}$ Thus psychosocial factors may be indirectly or directly linked to the processes that result in the perception of a visceral stimulus, and therefore consulters with functional GI disorders may differ from non-consulters. However, in the present study we found that significantly lower sensory thresholds occurred in non-patients with chronic relapsing upper abdominal symptoms. We conclude that visceral hyperalgesia is an important finding in $H$ pylori negative nonhealth care seeking subjects with a history of dyspepsia. This phenomenon of altered sensory function cannot be explained by referral bias as all subjects in our study were nonconsulters. Furthermore, specific personality traits did not explain the results; our study subjects were "normal" in terms of personality traits based on a validated measure for the local population.

It might be argued that our study may have inadvertently included subjects with a physical cause of symptoms because an upper GI endoscopy was not undertaken. However, in younger subjects with no alarm signals, no reflux symptoms, and a negative $H$ pylori breath test, a physical explanation for 
their symptoms is very unlikely. Indeed, insisting on an endoscopy could have resulted in selection bias because some non-patients would most likely have refused. The proportion of population based subjects reporting dyspepsia (24\%) was consistent with other population based studies in this region ${ }^{15}$ and elsewhere. ${ }^{16}$

In addition to lower pressure thresholds for first perception at baseline, subjects with a history of dyspeptic symptoms were characterised by a lack of increase in sensory thresholds after repeated phasic distension. In healthy patients, we observed a possible adaptive response to repeated mechanical distension (that is, gastric sensory thresholds increased). Previous studies in IBS patients ${ }^{27}$ have demonstrated a decrease in sensory thresholds following repeated mechanical colorectal stimulation (referred to as rectal hyperalgesia). Based on the available data, we speculate that both disturbed adaptive responses of the perception threshold and induction of visceral hyperalgesia following repeated mechanical stimulation contribute to the abnormalities in visceral sensory function observed in patients with functional GI disorders. It might well be that visceral hyperalgesia induced by repeated mechanical stimulation of the colon is a phenomena that occurs in IBS patients while a lack of adaptive responses in the stomach characterises patients with functional dyspepsia. Nevertheless, both may contribute to the observed differences between healthy subjects and patients with functional GI disorders.

A disturbance in endogenous pain modulation systems is a potential explanation for our findings. Based on previous animal studies, it appears most likely that mechanical stimulation activates descending corticospinal, bulbospinal, and spinal inhibitory systems. ${ }^{5}$ These mechanisms probably protect against the development of sensitisation or even increase visceral sensory thresholds but may be disturbed in functional dyspepsia. Afferents activating the putative descending inhibitory pathways are most likely mediated by the vagal nerve ${ }^{28}$ and vagal dysfunction is closely linked with visceral hyperalgesia in functional dyspepsia. ${ }^{29}$ In healthy asymptomatic subjects, activation of descending inhibitory pathways may prevent sensitisation (resulting in lowered sensory thresholds) while failure to activate these descending pathways may be responsible for the development of symptoms in patients with functional dyspepsia.

In the current study, we assessed pressure and calculated wall tension assuming a spherical shape of the gastric barostat device. Both measurements yielded significant differences between subjects with and without dyspeptic symptoms. It is widely assumed that the balloon in the stomach has a spherical shape $\mathrm{e}^{20}$ and this is also supported by $x$ ray studies we have conducted in the past. However, it is possible that the balloon may not always assume a spherical shape. Nevertheless, similar results were observed in the present study based on analysis of pressure and tension data, supporting the assumption that differences in sensory function between subjects with and without dyspeptic symptoms are real. In contrast, studies focusing on volume data alone may not yield accurate results; indeed, in our study differences in volume were not statistically significant.

The current study did not allow us to identify the precise causes of the disturbed adaptive changes in subjects with unexplained dyspepsia. However, we speculate that treatment of some patients with functional dyspepsia may require normalisation of the ability to respond appropriately to a sensitising stimulus rather than simply increasing sensory thresholds. This may account for the relatively disappointing results with visceral analgesics such as kappa opioid agonists in functional dyspepsia $^{30}$ despite increases in sensory thresholds to balloon distension after fedotozine. ${ }^{31}$

In summary, the present study demonstrates that alterations in gastric mechanosensory function are not limited to patients with functional dyspepsia but can also be found in dyspeptic subjects who have not sought health care. In addition, an adaptive increase in gastric mechanosensory thresholds may occur in healthy asymptomatic subjects but not in subjects with dyspepsia after repeated mechanical stimulation. The lack of an adaptive increase in thresholds for first perception after repeated mechanical stimulation may play a role in the pathophysiology of functional dyspepsia.

Supported by a grant from Deutsche Forschungsgemeinschaft, grant No Ho 1193/3-6.

1 Bradette M, Pare P, Douville P, et al. Visceral perception in health and functional dyspepsia. Crossover study of gastric distension with placebo and domperidone. Dig Dis Sci 1991;36:52-8

2 Mearin F, Cucala M, Azpiroz F, et al. The origin of symptoms on the brain-gut axis in functional dyspepsia. Gastroenterology 1991;101:999-1006.

3 Ritchie J. Pain from distension of the pelvic colon by inflating a ballon in the irritable bowel syndrome. Gut 1973;14: 125-32.

4 Whitehead WE, Holtkotter B, Enck P, et al. Tolerance of rectosigmoid distension in irritable bowel syndrome.

5 Mayer EA, Gebhart GF. Basic and clinical aspects of visceral hyperalgesia. Gastroenterology 1994;107:271-93.

6 Gisceral hyperalgesia. Gastroenterology 1994;107:271-93. Am Pain Soc f 1992;1:26-32.

7 Collins SM. Irritable bowel syndrome could be an inflammatory disorder. Eur $\mathcal{F}$ Gastroenterol Hepatol 1994;6: 478-82.

8 Silverman DHS, Munakata JA, Ennes H, et al. Regional cerebral activity in normal and pathological perception of visceral pain. Gastroenterology 1997;112:64-72.

9 Whitehead WE, Bosmajian L, Zorderman AB, et al. Symptoms of psychologic distress associated with irritable bowel syndrome. Comparison of community and medical clinic syndrome. Comparison of community and
samples. Gastroenterology 1988;95:709-14.

10 Drossman DA, Sandler RS, McKee DC, et al. Bowel patterns among subjects not seeking health care: use of a questionnaire to identify a population with bowel dysfuncquestionnaire to identify a population

11 Scarinci IC, McDonald Haile J, Bradley LA, et al. Altered pain perception and psychosocial features among women with gastrointestinal disorders and history of abuse: A preliminary model. Am f Med 1994;97:108-18.

12 Mertz H, Naliboff B, Munakata J, et al. Altered rectal perception is a biological marker of patients with the irritable bowel syndrome. Gastroenterology 1995;109:40-52.

13 Talley NJ, Phillips SF, Melton LJI, et al. A patient questionnaire to identify bowel disease. Ann Intern Med 1989;111: naire to

14 Holtmann G, Goebell H, Holtmann M, et al. Dyspepsia in healthy blood donors: pattern of symptoms and association with Helicobacter pylori. Dig Dis Sci 1994;39:1090-8.

15 Holtmann G, Goebell H, Talley NJ. Dyspepsia in consulters and non-consulters: prevalence, health-care seeking behav10ur and 24 . 
16 Talley NJ, Zinsmeister AR, Schleck CD, et al. Dyspepsia and dyspepsia subgroups: a population-based study. Gastroendyspepsia subgroups: a pop

17 Talley NJ, Silverstein MD, Agréus L, et al. Evaluation of dyspepsia. Gastroenterology 1998;114:582-95.

18 Jackson DN. Manual for the personality research form. Goshen: Research Psychologists Press, 1974.

19 Stumpf H, Angleiter A, Wieck T, et al. Deutscher personality research form (PRF). Göttingen: Verlag für Psychologie, 1985.

20 Distrutti E, Azpiroz F, Soldevilla A, et al. Gastric wall tension determines perception of gastric distension. Gastroenterology 1999;116:1035-42.

21 SAS Institute Inc. SAS/STAT User's Guide, Release 6.03. Cary, NC: SAS Institute Inc., 1988.

22 Holtmann G, Goebell H, Talley NJ. Impaired small intestinal peristaltic reflexes and sensory thresholds are independent functional disturbances in patients with chronic unexplained dyspepsia. Am $\mathcal{F}$ Gastroenterol 1996;91:48591 .

23 Bradette M, Delvaux M, Staumont G, et al. Evaluation of colonic sensory thresholds in IBS patients using a barostat: Definition of optimal conditions and comparison with healthy subjects. Dig Dis Sci 1994;39:449-57.

24 Whitehead WE, Crowell MD. Psychologic considerations in the irritable bowel syndrome. Gastroenterol Clin North Am 1991;20:249-67.
25 Zuckerman MJ, Guerra LG, Drossman DA, et al. Health-care-seeking behaviors related to bowel complaints: Hispanics versus non-Hispanic whites. Dig Dis Sci 1996;41: 77-82.

26 Talley NJ, Fung LH, Gilligan IJ, et al. Association of anxiety, neuroticism, and depression with dyspepsia of unknown cause. A case-control study. Gastroenterology 1986;90:88692.

27 Munakata J, Naliboff B, Harraf F, et al. Repetitive sigmoid stimulation induces rectal hyperalgesia in patients with irritable bowel syndrome. Gastroenterology 1997;112:55-63.

28 Randich A, Ren K, Gebhart GF. Electrical stimulation of cervical vagal afferents. II. Central relays for behavioral antinociception and arterial blood pressure decreases. $\mathcal{F}$ Neurophysiol 1990;64:1115-24.

29 Holtmann G, Goebell H, Jockenhövel F, et al. Altered vagal and intestinal mechano-sensory function in chronic unexplained dyspepsia. Gut 1998;42:501-6.

30 Read NW, Abitbol JL, Bardhan KD, et al. Efficacy and safety of the peripheral kappa agonist fedotozine versus placebo in the treatment of functional dyspepsia. Gut 1997;41:6648.

31 Coffin B, Bouhassira D, Chollet R, et al. Effect of the kappa agonist fedotozine on perception of gastric distension in healthy humans. Aliment Pharmacol Ther 1996;10:919-25. 